\title{
Ego-Centered Cognitive Social Structures of Close Personal Networks in the United States
}

\author{
Christopher Steven Marcum ${ }^{\dagger} \quad$ Jeffrey Lienert $\quad$ Megan Goldring \\ Jielu Lin Alicia Miggins \\ Hena Thakur Laura M Koehly \\ Sunmi Song
}

May 16, 2017

\begin{abstract}
Social network analysis is increasingly important in the social and behavioral sciences and has been employed to study a host of inter- and intra-personal social processes. One of the challenges researchers face in this area, however, is balancing the trade-offs between different modes of network measurement and study design. At one end of the spectrum, entirely ego-centered network designs facilitate access to a large, generalizable sample of the population but often lack details on the underlying network structure that embed each respondent. At the other end, whole-network designs offer fine details about the network structure but are costly and suffer from generalizability limitations. In this paper, we employ an ego-centered network sampling design that strikes a balance between these two cases by leveraging how individuals perceive their social worlds vis-a-vis respondent reports of their alter-alter ties. We describe a large sample of close personal networks where respondents informed on their perceptions of the ties between their alters on multiple types of relations. Specifically, we characterize the distribution of network statistics (size, density, and multiplexity) for over a thousand individual ego-centered cognitive networks drawn from a representative sample of the U.S. population. To our knowledge this is the first study to characterize the distribution of mental maps vis-a-vis perceived alter-alter relationships in this large of a sample of respondents involved in close personal networks. In doing so, we more clearly shed light on how Americans perceive the structure of their social worlds and provide an empirical case study in what we characterize as ego-centered cognitive social structures.
\end{abstract}

There is a large body of research showing that interpersonal ties and embeddedness within social network systems has important implications in one's mental and physical health (Thoits, 2011). Such value can be derived through many venues - connections with family, coworkers and friends, as well as within community organizations (Cattell, 2001). Moreover, it is not simply the mere existence of social ties that is important to health outcomes, 
but rather aspects of the structure and function of these network systems (Cattell, 2001; Kawachi et al., 2008). Notably, the majority of this research has used an ego-centered network approach to delineate how network structure and function are related to such outcomes. In large part, these inquiries are limited to structural characteristics that describe network composition, network size, and the type, or function, of resources exchanged within the social system. In some, perceived network density or perceived reciprocity in exchanged resources is captured, allowing for a broader inquiry of how network structure is related to health (Väänänen et al., 2005; Ashida and Heaney, 2008). While this research has provided much insight into the important role social relationships play in health, there are limitations in this work.

First, there are few examples of large, population-based samples that capture participants' perceptions of the social network systems within which they are embedded (Fischer, 1982). The majority of this research considers participants that are co-located within a particular geographic setting, is homogeneous with respect to age, gender, and race, or considers a limited set of relations and network structural characteristics (Christakis and Fowler, 2007; Ajrouch et al., 2001; Klovdahl et al., 1994; Berkman and Syme, 1979). As such, we have an incomplete view of how the adult population within the United States (U.S.) perceives their social worlds. Expanding our understanding of these perceived social systems has important implications for both research and practice. For example, much ego-centered network research employs narrowly-selected samples, which limits the generalizability of results to the population. A population-based sample would provide a set of reference distributions for ascertaining how self-selected samples differ from the population in terms of network structure and function. By so doing, one can evaluate the reach of potential insights gained from these smaller studies. Further, in practice, such a distribution represents gained knowledge of individuals' social environments across the lifecourse, allowing us to identify populations that might be particularly vulnerable to negative health outcomes based on the nature of their perceived network systems. 
Second, variability in sampling design, data collection approaches, and analysis strategies prevent a comprehensive and accurate understanding of these network characteristics and processes (Freeman and Thompson, 1989; Morrison, 2002; Shye et al., 1995). One commonly implemented approach is to deeply characterize a single network system, engaging multiple network members to provide their perceptions of their social ties. This approach provides information on how that network is structured and the patterns of resources exchanged among members. Using such a case study design to elicit whole, or nearly whole networks, provides considerable information about a given system but limits our ability to generalize observed associations across similar systems. At the other extreme, large numbers of respondents are sampled and asked to provide their perceptions of the local structure that surrounds them. Often, assessments of this local structure are limited to a small set of network functions, with a focus on the relationships between the participant informant and the network members that they enumerate. While network characteristics derived from such a design may be generalizable beyond the particular sample investigated, such an approach is often limited to measuring only a few structural properties due to the local network focus.

To address some of these limitations, the current paper has two primary aims. First, given the lack of consensus on how to collect, define, and analyze personal networks (Marsden, 1987), we provide definitional clarity through a brief review of different ego-centered network study designs commonly encountered in the literature. Second, we characterize the structure and function of a set of personal networks obtained from a population-based sample of U.S. adults. Specifically, we describe network composition and the distribution of network statistics derived from these personal networks, with a focus on size, density, multiplexity, and heterogeneity. The resulting information provides baseline metrics on these structural characteristics that can be used for imputation, simulation, and comparisons to non-representative samples. Our approach follows the pioneering efforts of the 1985 GSS Social Network module (Burt, 1984) but is implemented on a larger scale with improved cost-effectiveness by utilizing an existing representative consumer panel and an online net- 
work survey instrument.

\section{Past work on personal networks}

A substantial amount of effort has gone into estimating "who you know" networks, or the broadest social structure surrounding a given ego with the goal of ascertaining the upperbounds on social network size (Bernard et al., 1991). These networks are sometimes called contact networks, acquaintanceship networks, or personal networks. There is little consistency in the reported results across different studies. Network size, for example, ranges between 300 to 1500 alters (Bernard et al., 1990; Killworth et al., 2006; Zheng et al., 2006); this variation is likely the result of heterogeneity induced by variability in study designs and collection methods (which we discuss in the next section).

While an interesting research question, examining the relationship between network size, or the number of persons the ego informant knows, and some outcome does not consider the complexities of interpersonal processes that may influence human behavior and, ultimately, health and well-being. For example, the ties incident between egos and their respective alters are also widely heterogeneous in composition. Moreover, the quality of social relationships and the resources exchanged within these networks are also important, and some would argue that such qualitative and functional aspects of these networks are more important than merely the number of persons an individual knows (Roberts and Dunbar, 2011; Marsden, 1987; McPherson et al., 2006; Roberts et al., 2009a). Indeed, there is a robust literature investigating network members' social support functions (Bernard et al., 1991; Milardo, 1992). This literature focuses on more intimate social relationships with the assumption that (or question of whether) these close ties are intrinsically more important than other types of ties in the lives of egos. The first large-scale study to add this level of specificity was the General Social Survey, which limited the type of relationship enumerated to delve further into the structure and distribution of pre-specified close ties. As part of the General Social Survey, Burt (1984) collected the "intimate ties" surrounding an ego using a multiple-item 
measurement approach. The General Social Survey assessed the quality of interpersonal ties (i.e., "feel especially close to") and the function of those ties (i.e. "discuss personal matters") and the frequency of interaction (i.e., "met with frequently") in their ego-centered network elicitation. Consistent with the General Social Survey approach, we propose that personal networks can have a broader definition than simply acquaintanceship or contact networks (i.e., "who you know"); such networks can include a myriad of relational ties between egos and their network members and can be constrained to specific role relations (i.e., kinship ties) or other close personal ties.

Still, Burt's (1984) evidence on close personal networks highlights that an incredible amount of variability exists around the way in which study design, in particular enumeration questions, can influence the operationalization of a 'close alter.' For example, Campbell and Lee (1991) empirically illustrated the influence of question-type on the size and structure of close networks. This heterogeneity was further demonstrated in the context of 'strong ties' (Smith, 2012), six different types of 'close' network ties (such as core discussion members and kin) (Smith et al., 2015), and slight alterations in the wording in close alter enumeration questions (Straits, 2000). As well, when considering functional aspects of the network system, Van der Poel (1993) asserts the superiority of 'exchange-based' name generators by describing the distribution of close networks across 10 different question types. The prevailing finding from this line of work is that the size of the network depends on the nature of the namegenerator: the closer the relationship elicited on the name generator the smaller the resulting network.

Despite the variability across study designs, we have gained insight from this literature with regards to the distribution of network statistics for personal networks, including acquaintanceship networks and close relationship networks. For example, networks that focus on close personal ties involved in everything from communication to social support have been reported to range in size, on average, between 2 and 9 alters(Van der Poel, 1993; McPherson et al., 2006; Smith et al., 2015) — though studies measuring the size of acquaintanceship 
networks report much higher numbers as we mentioned above. Thus, by conditioning on the type of relationships (here, broadly defined by some metric of closeness), the magnitude in network size is greatly reduced. These studies emphasize the importance of defining the type of network enumerated whether distinguishing qualitative aspects of interpersonal ties or the resources exchanged between egos and alters. Notably, many studies have also shown the importance of ego attributes in explaining the distribution of network size, density, and connectivity (Dunbar and Spoors, 1995; Roberts et al., 2009b; van den Berg et al., 2009).

Thus, the heterogeneity in name generators and variability in informant attributes used across studies can result in significant differences in network structural characteristics. Here, we focused primarily on one type ego-centered network design - that is, personal networks comprised of interpersonal ties between an ego-informant and their network members. There are a number of different ego-centered designs used to measure networks and each can significantly impact the distribution of network characteristics describing assessed networks. We now describe an organizing framework for ego-centered network designs with the goal of providing common terminology for the field and clarifying the benefits and limitations of each.

\section{Ego-centered designs}

One of the primary challenges to research on personal networks is the absence of a lingua franca across disciplines to describe different approaches to network study design. What one study may call personal networks another might call ego-centered and yet another might use the term egocentric. The canonical text on the subject, Wasserman and Faust (1994), defines a network as a set of actors and the relationships between them. They go on to define ego-centered networks as those consisting 'of a focal actor, termed ego, a set of alters who have ties to ego, and measurements on the ties among these alters' (pp.42). Thus, if we rely on this definition, there is no distinction between ego-centered and ego-centric as they both refer to anchoring the alter inclusion criteria on a particular ego (or focal actor/site/node). 
Notably, personal networks consider the social world surrounding a given ego, wherein the ego and alters are human actors.

Social network data collection can be described along a dimensional axis ranging from completely ego-centered to whole network (or socio-centric) study designs. In most applications, all designs along this axis rely on ego reports of interpersonal ties. These network assessments commonly engage human informants who provide their perceptions of social relations among network members, whether these perceptions are limited to relationships between the informant and other network members or consider perceptions of connections among members to whom the informant is not directly tied. The theoretical underpinnings of each type of these ego-centered designs are described in detail elsewhere (Marsden, 2005). Moving beyond the theoretical implications of each sampling design, researchers must also consider the logistical and structural benefits of each technique (Marsden, 1990). While entirely ego-centered networks tend to be easily collected and economically feasible, sociocentric networks provide richer information with respect to network structure.

Four archetypal variations of ego-centered network design are found in the literature include. Here, we refer to these variations as: 1) entirely ego-centered (EEC); 2) ego-centered cognitive social structure (ECSS); 3) multi-informant ego-centered (MIEC); and, 4) multiinformant cognitive social structure (CSS). These variations are depicted in Figure 1 and represent a series of trade-offs that balance cost (in terms of both respondent burden and price), network data complexity, and network accuracy.

In the first case, an EEC design collects no alter-alter ties. Egos enumerate their alters using a name-generator (or another standard approach as on a roster) and report only on their own ties with those alters. This is clearly the most cost-effective method of gathering network data and can be easily deployed using standard survey methodology and implemented on a large scale; however, the EEC design comes with a trade-off in lower measurable network structure and potentially, more informant bias. Additionally, the boundaries of the network are arbitrarily determined by the enumerator - a limitation described by Laumann et al. 
Figure 1: Examples of Ego-Centered Network Study Designs

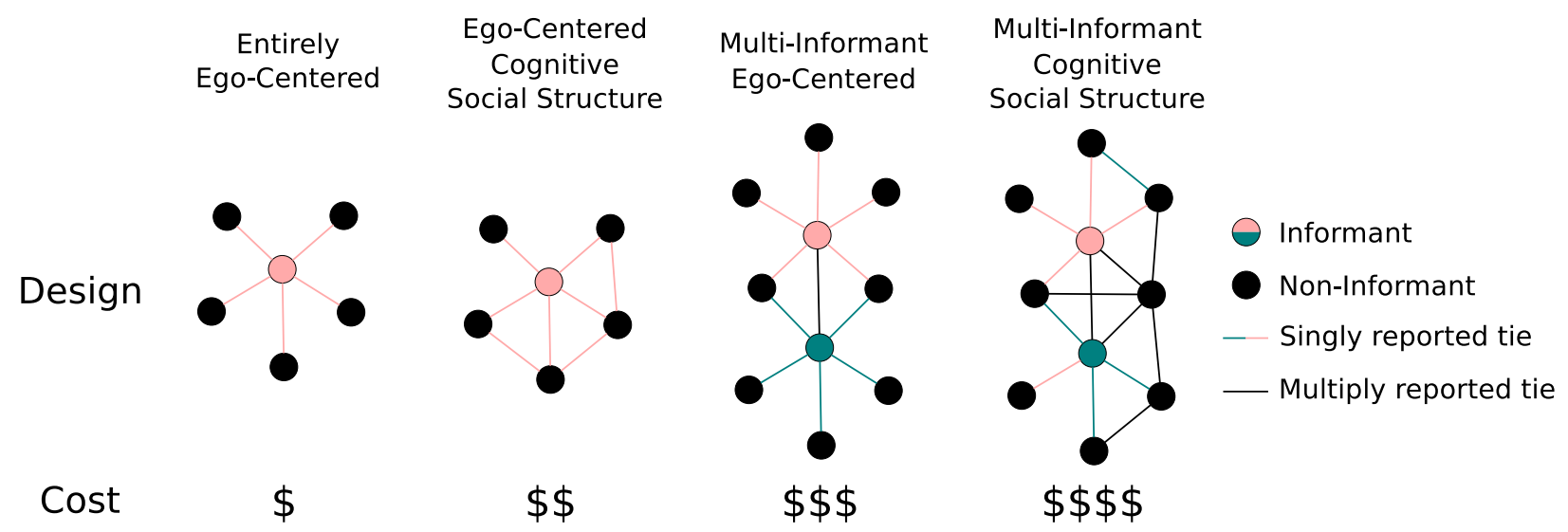

(1989). This approach is optimal when the research question seeks to explore variation in features of personal networks that can be aggregated nearly independently to the level of the ego, such as network size, local density, or composition (Roberts et al., 2009b; Omalley et al., 2012).

When a balance between having complex structure with low cost must be optimized, then an obvious extension to the EEC is an ego-centered approach that captures perceptions of alter-alter ties in addition to ego-alter ties - the ECSS design. Like EEC, this design results in a personal network, can be implemented on a large scale, and carries the limitation that the network boundary is determined by the sole enumerator/informant. This was famously done by the 1985 General Social Survey (Burt, 1984), though to reduce respondent burden and probable burnout via survey fatigue, the instrument limited alter-alter tie collection to the first five enumerants. Using a similar approach, McCarty et al. (2007) showed that burnout may not be as substantial a problem as suspected. We might characterize the ECSS as one slice through a cognitive social structure (CSS).

When some boundary can be drawn on the network (say, a family, or even the focal actor's alter set as in respondent-driven and snowball sampling), then a more costly but more structurally complex approach entails recruiting multiple-egos from within those boundaries who would independently enumerate their alters. This MIEC design may give rise to richer 
network data than an EEC design as it captures mutual ties and the number of shared partners between informants can be calculated. The design is not well-suited for large-scale implementation, though several studies using this design have been conducted (Koehly et al., 2015; 2003; Newman, 2003). Of note, this MIEC design results in socio-centric, or whole network, design when all members within the network bounds are ego-informants.

Perhaps the most well-known CSS design is that of Krackhardt (1987), which captures whole (or socio-centric) networks by taking measurements about the perceptions of ties from all members of a network within a well-defined boundary. In that seminal paper, the cognitive social structure is defined by $\mathbf{X}=x_{i j k}$, where $i$ is the "sender" of the relation on network $\mathbf{X}$, " $j$ " is the "receiver" of the relation, and " $k$ " is the "perceiver" (or observer/informant) of the relation from " $i$ " to " $j "$. The informants may be embedded within the network or exogenous. Thus, Krackhardt's design may be considered a special case of a general multiinformant CSS (MICSS), where either a sample or census of informants can be drawn from within those bounds. Obviously, this approach is very costly and not scalable in most cases, but yields very rich data.

The cognitive social structure approach, whether an ego-centered or multi-informant design, has been used to learn about the structural characteristics of people's social worlds. The advantage of such an approach allows for description of structural characteristics like density and triad structure (Wellman, 1979; Fischer, 1982; Burt, 1984; Boster et al., 1987; Neal, 2008). The ego-centered cognitive social structure design can be used to reveal important information about how individuals understand the context of their social world by describing perceived ego-alter and alter-alter relationships. However, there are few studies that have large-scale representation at the population level and lack appropriate empirical reference distributions for comparison. This is a gap that we now turn to address in our empirical example using an ECSS design. 


\section{Methods}

The purpose of our study was to describe the distribution of network characteristics related to the composition and structure of a large-scale representative sample of ego-centered cognitive networks from the US population. In particular, we focus on ego's perceptions of relationships between alters who have close interpersonal ties to egos. Considering the amount of heterogeneity in close personal networks, we choose to leverage the relevance of kin in our network enumeration technique, whereby we prime participants to think first of kin and then of important alters that are as close as kin (Roberts and Dunbar, 2011; Roberts et al., 2009a; McPherson et al., 2006; McCarty et al., 1997; Marsden, 1987). We employed an ego-centered cognitive social structure study design (ECSS). In this section, we describe the study design and sample that comprise our final dataset.

\section{Study Design}

All data were collected via an online survey using an ECSS design. In addition to respondent background characteristics (age, marital status, gender, etc), the network elicitation instrument asked respondents to list their alters and, for each alter, alter background characteristics, information about their relationship with those alters (i.e., ego-alter ties), and information about the relationships between alters (i.e., alter-alter ties). The specific instrument for the latter is discussed in detail below.

Researchers employing similar designs typically limit the number of alters an ego may enumerate - such as the Wellman (1979) East York study that asked ego about alter-alter ties among only six close confidants per interviewed ego. Such a constraint on the number of alters is usually implemented to reduce participant burden. In comparison, free-responses reveal important underlying biases that expose perceptions of social ties, while they are limited in that the boundary specification problem is left up to the respondent to resolve (Laumann et al., 1989). At the same time, we also recognize that participant burden is 
a concern for study design because the number of alter-alter relationships increases nearly quadratically with network size (McCarty et al., 2007). Therefore, for the current study, we took a three-part strategy to reducing the number of alter-alter pairs on which respondents report their perceptions to help alleviate potential burden. First, rather than limiting the number of enumerants, we used highly specific network enumerators that were designed to elicit small personal networks. Specifically, respondents were prompted with two enumerators eliciting immediate family ties and anyone else that they consider to be important to them (each, with examples):

Immediate family: We'd like learn about people that are important to you. First, please list all members of your family of origin (parents and siblings) and your nuclear family (spouse/partner and children). We'll ask about other important people later.

Other important people: Now we'd like you to think about other people that are important to you that are not members of your family of origin or nuclear family. Please list other people that are important to you, such as extended family members (your grandmother, grandchildren, cousins, etc.), family through marriage (mother-in-law, brother-in-law), friends, and coworkers.

Second, as part of the alter attribute module, we asked egos to indicate whether or not each listed alter was presently living. Any deceased alters were automatically removed from the list of dyads. Third, we asked a conditioning question - "Do A and B know each other?", where A and B were replaced with the names of two listed alters - to generate a mental map of acquaintanceship among each ego's set of alters. For each dyad, if an ego indicated "yes" to this conditioning question, then they were presented with additional network questions about the pair later in the survey; if they answered "no" or "don't know" then the dyad was dropped from further interrogation. By adding these conditions, we reduced the expected burden on our respondents when requesting alter-alter information. 
When two network members were indicated to know one another by the who-knows-who conditioning question, we also asked a series of additional network questions that tap into aspects of relationships expected to be active in close personal networks (Koehly and Marcum, 2016). These included four binary directed relationships:1) who seeks advice from whom, 2) who consoles whom when their upset, 3) who discusses their health with whom, and, 4) who shares their feelings with whom. Two additional questions about the respondent's perspective on the extent of conflict and closeness between two network (non-directed 7 point scales) were also asked. These were asked for all valid ego-alter and alter-alter ties.

\section{Online Consumer Panel}

We used a customized online platform to administer our ECSS assessment to an existing consumer panel engaged by QSample ${ }^{\mathrm{TM}}$, which allowed us to match the respondent pool to be representative of English speaking adults on age, gender, race and ethnicity in the US population. Most respondents in this pool are accustomed to taking online surveys (and are thus, representative of that group), though the vendor reports ours was the first network study to collect alter-alter ties from the pool.

For egos' background characteristics we collected age, ethnicity, gender, highest educational attainment, marital status, race, and zip-code of residence. Covariate information for alters included age (or deceased status otherwise), gender, and type of relationship to ego.

A total of 4,467 individuals responded to the survey. About 20\% ( $\mathrm{n}=883$ ) were ineligible to participate based on our inclusion criteria (e.g., English speaking, above 18 years of age, and willing to provide information on at least one alter) and were then excluded from subsequent survey questions. Of all 3,584 respondents, 1,875 completed the entire questionnaire. The remaining 1,706 completed portions of the questionnaire and elected to quit the survey. The final effective response rate is $53 \%$. Women have slightly higher completion rate than men, and there is virtually no difference in completion rate by other demographic characteristics. In the analyses described below, we additionally dropped 51 responses due 
to missing data (for a final sample size of 1824).

We must report two important notes about the coding of the ego-alter relationships. First, the "other non-relative" category is a hodgepodge of very low frequency response options including other acquaintances, co-workers, and medical professionals etc. Second, the "grandchild" response option to the relationship type was inadvertently pooled with "other relatives" in the survey database. Therefore, we imputed "grandchild" by inductive reasoning based on the empirical distribution of grandparents and grandchildren from U.S.

Census data jointly with the age difference between the ego-alter dyad. If the age difference was greater than or equal to the minimum age difference found in the 2010 Census data on grandparent headed households (roughly 35 years), then we flipped a biased coin to decide whether that "other relative" should be coded as a grandchild with probability 0.3 (the rate of grandparenthood from the 2010 Census). This procedure resulted in $20 \%$ of the 1,736 originally coded "other relatives" being imputed as grandchildren $(\mathrm{n}=348)$.

\section{Results}

We begin by describing the attributes of the egos and their alters. Table 1 reports descriptive statistics on covariates (as frequencies and percent frequencies). Respondents comprising our final sample provided information on more than 16,000 alters. About half of egos and half of alters were female. Most respondents and their alters were middle-aged (between the ages of 50 and 65). Our sample of egos was about $80 \%$ white, $10 \%$ Hispanic, most had at least a college education, and most were married (about 50\%). The descriptive results on age, gender, and race of the respondents (egos) lend confidence to the representative nature of the online panel sample. Additionally, as illustrated by the choropleth of respondent zip-codes plotted in Figure 2, our sample was well-distributed geographically across the United States with representatives from every state including Alaska and Hawaii.

Our first set of network descriptive statistics focus on ego-alter ties. Figure 3 plots 
Table 1: Descriptive Statistics of the Sample

\begin{tabular}{|c|c|c|}
\hline & Egos & Alters \\
\hline $\mathrm{N}$ & 1824 & 16515 \\
\hline Female & 0.5400 & 0.5200 \\
\hline \multicolumn{3}{|l|}{ Age } \\
\hline $0-4$ & - & 0.0280 \\
\hline $5-9$ & - & 0.0296 \\
\hline $10-14$ & - & 0.0322 \\
\hline $15-19$ & 0.0159 & 0.0363 \\
\hline $20-24$ & 0.0482 & 0.0516 \\
\hline $25-29$ & 0.0855 & 0.0664 \\
\hline $30-34$ & 0.0861 & 0.0634 \\
\hline $35-39$ & 0.0762 & 0.0589 \\
\hline $40-44$ & 0.0828 & 0.0603 \\
\hline $45-49$ & 0.0811 & 0.0638 \\
\hline $50-54$ & 0.1075 & 0.0780 \\
\hline $55-59$ & 0.1118 & 0.0873 \\
\hline $60-64$ & 0.1162 & 0.0767 \\
\hline $65-69$ & 0.0998 & 0.0777 \\
\hline $70-74$ & 0.0493 & 0.0539 \\
\hline $75-79$ & 0.0269 & 0.0391 \\
\hline $80-84$ & 0.0060 & 0.0291 \\
\hline $85-89$ & 0.0055 & 0.0234 \\
\hline $90-94$ & 0.0011 & 0.0142 \\
\hline $95-99$ & - & 0.0059 \\
\hline $100+$ & - & 0.0066 \\
\hline deceased & - & 0.0140 \\
\hline Don't know & - & 0.0037 \\
\hline \multicolumn{3}{|l|}{ Race } \\
\hline Asian & 0.0530 & - \\
\hline Black or African American & 0.1160 & - \\
\hline Indian or Alaska Native & 0.0160 & - \\
\hline Native Hawaiian or other Pacific Islander & 0.0050 & - \\
\hline White & 0.7980 & - \\
\hline Don't know & 0.0120 & - \\
\hline Ethnicity & & \\
\hline Hispanic & 0.0943 & - \\
\hline Non-Hispanic & 0.9035 & - \\
\hline \multirow{2}{*}{\multicolumn{3}{|c|}{ Educational Attainment }} \\
\hline & & \\
\hline Less than 8 th grade & 0.0005 & - \\
\hline 8th grade & 0.0027 & - \\
\hline Some high school (no diploma or GED equivalent) & 0.0192 & - \\
\hline High school diploma or GED equivalent & 0.1826 & - \\
\hline Some college (no degree) & 0.2478 & - \\
\hline Technical or Associate degree & 0.1338 & - \\
\hline Bachelors degree & 0.2834 & - \\
\hline Masters degree & 0.1003 & - \\
\hline \multirow{2}{*}{\multicolumn{3}{|c|}{ Professional degree }} \\
\hline & & \\
\hline Divorced & 0.1146 & - \\
\hline Domestic Partnership & 0.0479 & - \\
\hline Married & 0.4869 & - \\
\hline Separated & 0.0105 & - \\
\hline Single & 0.2981 & - \\
\hline Widowed & 0.0390 & - \\
\hline Prefer not to say & 0.0030 & - \\
\hline
\end{tabular}


Figure 2: Choropleth of the Geographic Distribution of Respondents in the United States

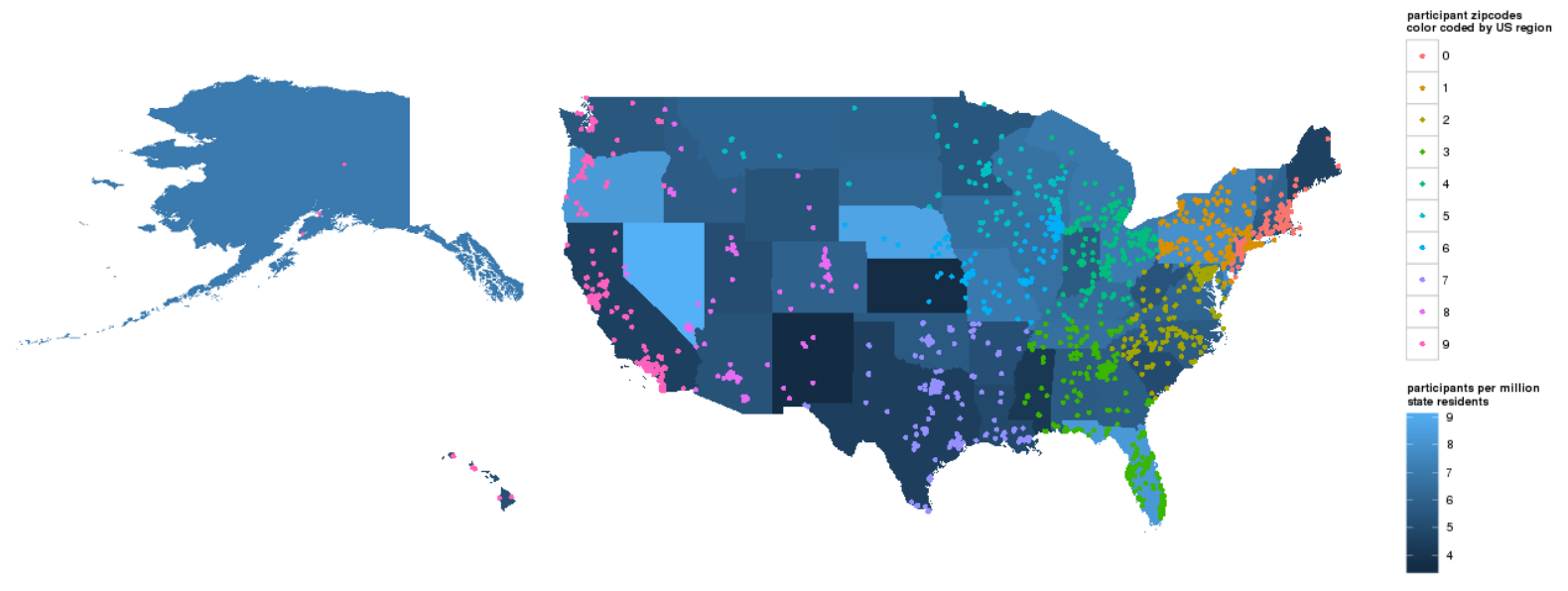

Each dot represents at least one respondent from a particular zip-code in the United States. Dots are colored by US postal code region to illustrate the relative regional distribution of the respondents. State polygons are shaded proportional to the population-adjusted coverage in that state (lighter blue means greater relative coverage).

the frequency distribution of the number of alters enumerated by the respondents. Each bar represents the total number of respondents ( $\mathrm{n}=1824$ egos) on the $\mathrm{y}$-axis enumerating a number of alters on the x-axis. The figure may be characterized as a population level egocentered degree distribution of close personal contacts. The median degree was about 9 alters (sd=6.5). Contributing to the heavy right tail, $90 \%$ of egos nominated fewer than 17 alters with a few enumerating more than 30 (the exact count is also reported in Table 2). Maximum likelihood was used to fit these data to several counting process distributions (Geometric, Negative-Binomial, Poisson, Poisson-Mixture) a la Handcock and Jones (2004). The results reveal that the ego-centered network size distribution is best described as $\sim \operatorname{Neg} \operatorname{Bin}(3,9)$ with Kolmogorov-Smirnov statistic $k=0.023(p<0.368)$, which is a very good fit.

Figure 4 illustrates the relative composition of types of alter relationships pooled across all egos. As expected by design, the majority of alters $(80 \%)$ were kin, with siblings representing the most frequent type of relation. The balance of the networks were comprised by nonkin, with nearly all of the remaining $20 \%$ non-kin encompassed by the friendship category. Fewer than 50 egos reported only a single alter in their network (and those were most 
Figure 3: Frequency Distribution of the Number of Alters Enumerated by Respondents

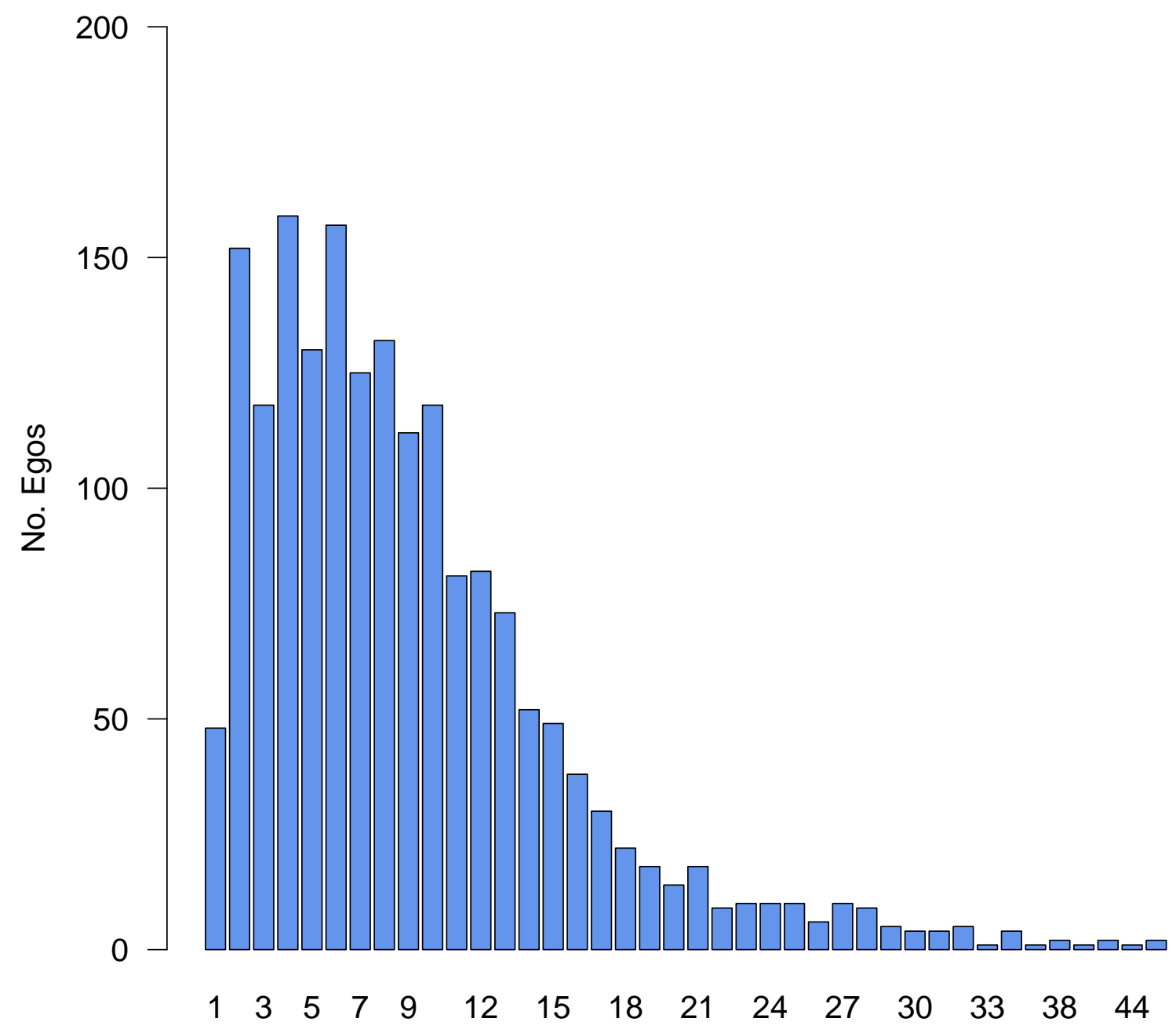

No. Alters

Each bar represents the total number of respondents $(n=1824$ egos) on the $y$-axis enumerating a number of alters on the x-axis. The figure may be characterized as an ego-centered degree distribution of close personal contacts. The median degree was about 9 alters $(\mathrm{sd}=6.5)$. Contributing to the heavy right tail, $90 \%$ of egos nominated fewer than 17 alters. Very few egos reported only a single close contact (and those were equally most likely to be a friend, a parent, or a spouse at $22 \%$ ). 
likely to be a friend, a parent, or a spouse at around $22 \%$ each). We also note that the enumeration questions were successful at distinguishing immediate family of origin from everyone else - with all children, parents, siblings, and spouse-partners listed in response to the first enumeration question and all others listed in response to the second question.

Moving onto network structure, we report a set of results on ego-alter, as well as alteralter, data. Roughly $8 \%(\mathrm{n}=152)$ of our sample consisted of ego-stars in the who-knows-who network (no reported alter-alter ties on any relation) with 121 of these stars being egocentered networks smaller than size 3 (sans egos). Two of the ego-stars consisted of 50 alters. There was no difference in the relative frequency of relationship types between egostars and other networks, though ego-stars were more likely to be provided by men than women $(p<0.004)$. Beyond that, for each network size, we observed great heterogeneity in network density and structure. Table 2 reports the frequency distribution of network size illustrated in Figure 3 along with the average network densities for each network size and type of relation conditional on the who-knows-who network. As expected, these networks tend to be relatively dense, though they are far from being complete. It appears that the five networks larger than size 39 provide very little useful data, which is likely the result of respondent burnout (though 1 respondent with a network size of 44 appears to have provided information on all alter-alter questions).

Figure 5 presents four selected networks (sans-ego) of various sizes (5, 6, 13, and 14) and demonstrates structural heterogeneity both within and between networks. The first row represents who-knows-who from egos' perspectives on the members of their personal networks; edges in all other subsequent rows measured multiplex ties conditional on this first relation. Between networks, there is evidence of structural heterogeneity in terms of size, density, and other local features (bridges, pendants, triangles, etc) across all relations. Within networks, that is across relations, heterogeneity is less pronounced, with a high degree of apparent multiplexity across relations in these examples. These results warrant a more formal examination of structural heterogeneity and multiplexity in the dataset, to which we 
Figure 4: Percent Frequency Composition by Relationship Type

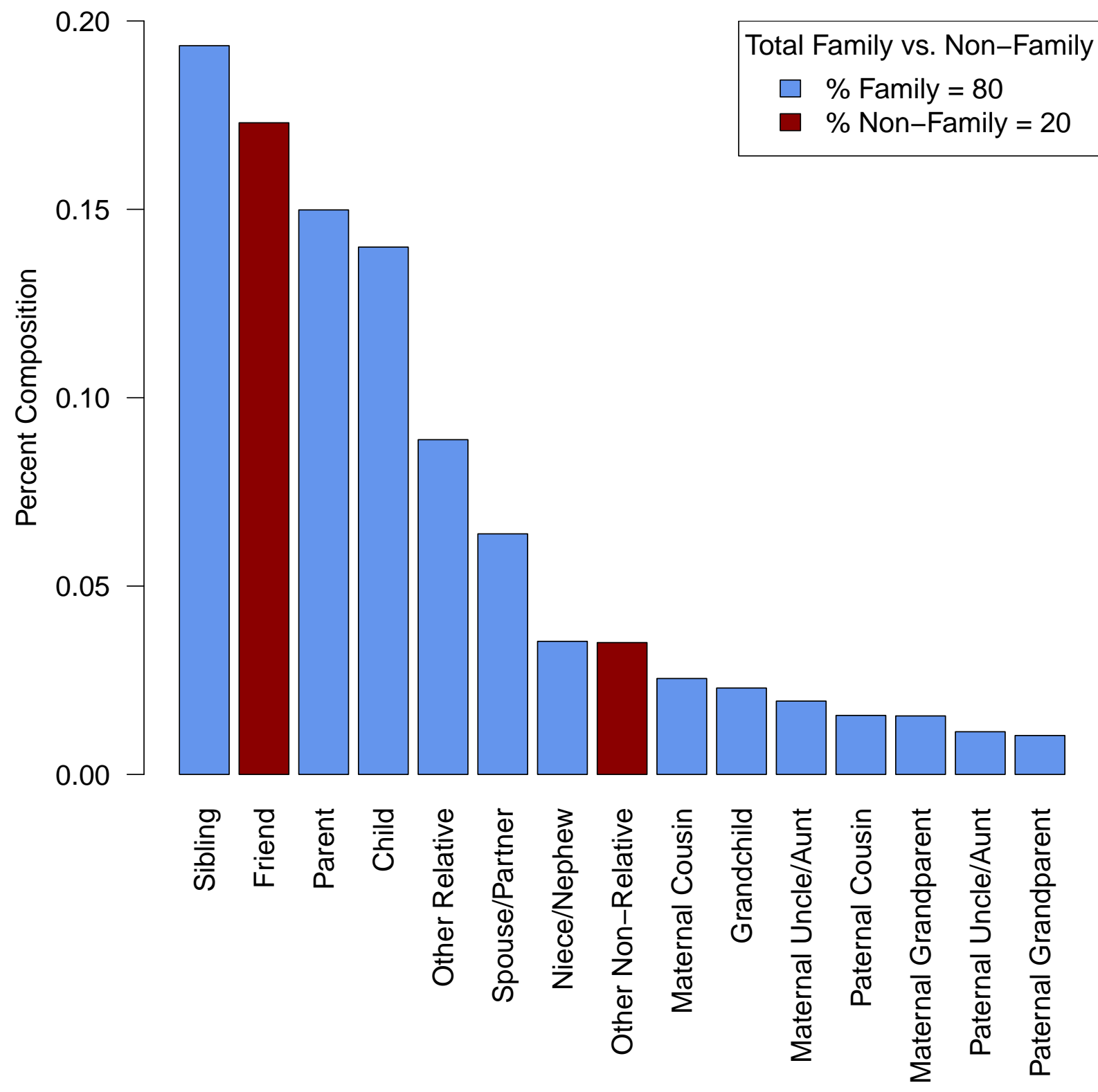

Each bar represents the fraction of all alters enumerated of a particular relationship type (i.e., to ego). The darker red bars indicate non-family types of relationships, which comprise about $20 \%$ of all enumerated alters. 
Table 2: Average Network Density for Who-Knows-Who, Consolation, Advice Seeking, Health Discussion, Share Feelings, Closeness, and Conflict Networks

\begin{tabular}{|c|c|c|c|c|c|c|c|c|}
\hline \multirow[b]{2}{*}{ Network Size } & \multirow[b]{2}{*}{$\mathrm{N}$} & \multicolumn{7}{|c|}{ Network Density } \\
\hline & & $\mathrm{WkW}$ & Console & Advice & Health & Feelings & Closeness $^{\dagger}$ & Conflict $^{\dagger}$ \\
\hline 1 & 48 & & & & & & & \\
\hline 2 & 152 & 0.684 & 0.638 & 0.589 & 0.647 & 0.704 & 0.221 & 0.217 \\
\hline 3 & 118 & 0.638 & 0.562 & 0.504 & 0.524 & 0.640 & 0.322 & 0.234 \\
\hline 4 & 159 & 0.677 & 0.648 & 0.581 & 0.623 & 0.690 & 0.431 & 0.237 \\
\hline 5 & 130 & 0.619 & 0.592 & 0.520 & 0.578 & 0.641 & 0.429 & 0.238 \\
\hline 6 & 157 & 0.594 & 0.596 & 0.526 & 0.573 & 0.646 & 0.449 & 0.254 \\
\hline 7 & 125 & 0.603 & 0.579 & 0.491 & 0.549 & 0.600 & 0.483 & 0.266 \\
\hline 8 & 132 & 0.519 & 0.568 & 0.496 & 0.534 & 0.609 & 0.479 & 0.233 \\
\hline 9 & 112 & 0.502 & 0.601 & 0.516 & 0.561 & 0.636 & 0.483 & 0.237 \\
\hline 10 & 118 & 0.487 & 0.575 & 0.490 & 0.543 & 0.638 & 0.475 & 0.243 \\
\hline 11 & 81 & 0.527 & 0.624 & 0.513 & 0.564 & 0.688 & 0.530 & 0.237 \\
\hline 12 & 82 & 0.500 & 0.569 & 0.453 & 0.516 & 0.607 & 0.513 & 0.233 \\
\hline 13 & 73 & 0.505 & 0.633 & 0.501 & 0.588 & 0.651 & 0.530 & 0.225 \\
\hline 14 & 52 & 0.459 & 0.591 & 0.472 & 0.501 & 0.626 & 0.515 & 0.224 \\
\hline 15 & 49 & 0.463 & 0.629 & 0.495 & 0.539 & 0.674 & 0.516 & 0.245 \\
\hline 16 & 38 & 0.442 & 0.644 & 0.554 & 0.634 & 0.692 & 0.543 & 0.225 \\
\hline 17 & 30 & 0.517 & 0.681 & 0.509 & 0.598 & 0.735 & 0.598 & 0.250 \\
\hline 18 & 22 & 0.444 & 0.721 & 0.534 & 0.559 & 0.670 & 0.535 & 0.241 \\
\hline 19 & 18 & 0.378 & 0.652 & 0.446 & 0.603 & 0.712 & 0.526 & 0.220 \\
\hline 20 & 14 & 0.362 & 0.618 & 0.441 & 0.470 & 0.605 & 0.555 & 0.235 \\
\hline 21 & 18 & 0.369 & 0.688 & 0.491 & 0.627 & 0.715 & 0.540 & 0.233 \\
\hline 22 & 9 & 0.382 & 0.694 & 0.414 & 0.589 & 0.603 & 0.557 & 0.226 \\
\hline 23 & 10 & 0.342 & 0.808 & 0.541 & 0.641 & 0.738 & 0.509 & 0.240 \\
\hline 24 & 10 & 0.352 & 0.544 & 0.458 & 0.497 & 0.581 & 0.589 & 0.248 \\
\hline 25 & 10 & 0.369 & 0.669 & 0.536 & 0.536 & 0.643 & 0.487 & 0.248 \\
\hline 26 & 6 & 0.170 & 0.718 & 0.508 & 0.559 & 0.846 & 0.438 & 0.136 \\
\hline 27 & 10 & 0.341 & 0.633 & 0.502 & 0.545 & 0.657 & 0.514 & 0.212 \\
\hline 28 & 9 & 0.077 & 0.639 & 0.593 & 0.666 & 0.688 & 0.259 & 0.157 \\
\hline 29 & 5 & 0.147 & 0.783 & 0.634 & 0.636 & 0.745 & 0.236 & 0.158 \\
\hline 30 & 4 & 0.049 & 0.686 & 0.872 & 0.650 & 0.917 & 0.251 & 0.093 \\
\hline 31 & 4 & 0.013 & 0.839 & 0.682 & 0.689 & 0.796 & 0.211 & 0.114 \\
\hline 32 & 5 & 0.026 & 0.991 & 0.794 & 0.791 & 0.920 & 0.317 & 0.080 \\
\hline 33 & 1 & 0.053 & 0.953 & 0.660 & 0.562 & 0.884 & 0.515 & 0.141 \\
\hline 34 & 4 & 0.013 & 0.739 & 0.720 & 0.720 & 0.739 & 0.150 & 0.049 \\
\hline 35 & 1 & 0.025 & 0.750 & 0.357 & 0.545 & 0.714 & 0.146 & 0.073 \\
\hline 38 & 2 & 0.102 & 0.747 & 0.576 & 0.552 & 0.561 & 0.535 & 0.165 \\
\hline 39 & 1 & 0.047 & 0.250 & 0.250 & 0.250 & 0.250 & 0.128 & 0.079 \\
\hline 40 & 2 & 0.013 & & & & & & \\
\hline 44 & 1 & 0.006 & 0.333 & 0.417 & 0.583 & 0.583 & 0.173 & 0.061 \\
\hline 50 & 2 & 0.000 & & & & & & \\
\hline
\end{tabular}


now turn.

To evaluate the extent of structural heterogeneity across our sample we calculated graph structural correlations (Butts and Carley, 2005) within each set of who-knows-who networks of a particular size and density from networks sized 4 to 20 (sans egos) using simulated annealing (networks larger than 20 pushed the limits of our computational ability to find proper structural correlations, see Butts and Carley (2005) for a discussion of such limitations). Structural correlations describe the maximum graph correlation between all accessible permutations of pairs of unlabeled graphs. When these correlations are 1, it means that some permutation about the rows and columns of the network adjacency matrices exists that transforms one network into another. Thus, it is a measure of the extent of unlabeled structural heterogeneity between two networks of the same size and density.

Table 3 reports the percent of networks of a particular size that were structurally unique along with the associated average per-density structural correlations. On average, structural correlations were high $(\hat{\rho}=0.667)$. Due to fewer opportunities for variations in network structure, smaller networks had a lower share of structurally unique networks. The largest networks in this analysis (the 14 networks of size 20) were all structurally unique (and thus, no structural correlation was possible because we conditioned on density). To examine the variation within a particularly likely scenario, we plot the density frequency distribution for networks of median size (9) together with every structurally unique network in that subset in Figure 6. The top bars represent the network density frequency distribution of all networks of size 9 in the dataset. The bottom networks represent all observations of the structurally unique networks at each network density cut-off. The leftmost segment thus represents the single-edge network and the rightmost segment represents the complete network. The figure demonstrates that, despite average high structural correlation and density as we reported above, there is substantial structural variation across networks of this size.

Finally, we evaluate average within-network heterogeneity by examining the multiplexity of the four binary directed relations: who consoles whom; who seeks whom for advice; who 
Figure 5: Example of Four Ego-Centered Cognitive Networks (sans-ego) and Relationships

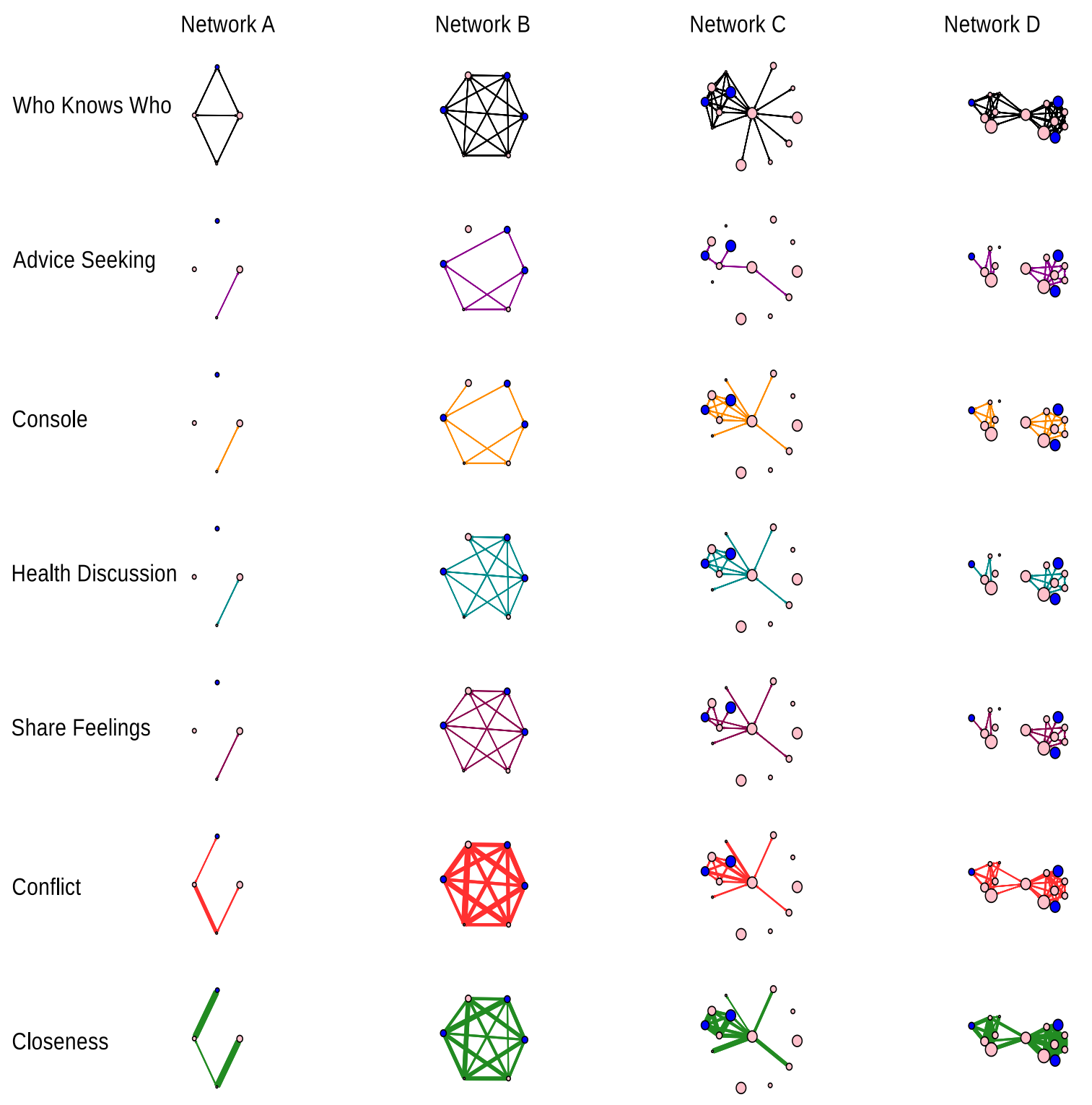

The first row of this figure illustrates four "Who-Knows-Who" ego-centered cognitive networks. Egos were removed to highlight the heterogeneity of perceived alter-alter relationships. The second through seventh rows depict the six measured alter-alter attributes within these networks. The relative quantitative measurements of the closeness and conflict frequency attributes are reflected by the alter-alter tie widths. Blue nodes are male alters, pink nodes are female alters, and the relative node sizes represent alter age 


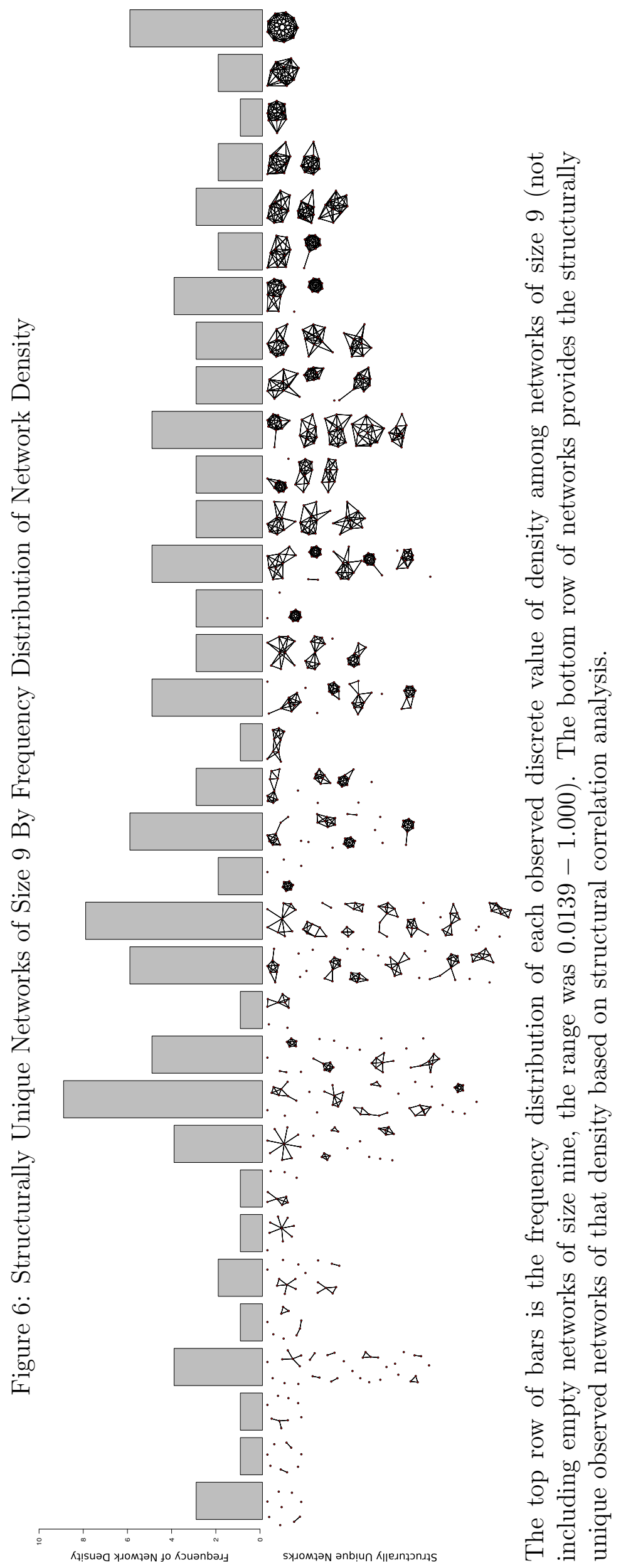


Table 3: Structural Uniqueness for Networks Sizes 4 through 20 of the Same Density

\begin{tabular}{ccc}
\hline \hline Network Size & Fraction Structurally Unique & Mean Structural Correlation \\
\hline 4 & 0.045 & 0.831 \\
5 & 0.091 & 0.795 \\
6 & 0.115 & 0.789 \\
7 & 0.148 & 0.683 \\
8 & 0.162 & 0.699 \\
9 & 0.232 & 0.710 \\
10 & 0.288 & 0.667 \\
11 & 0.383 & 0.696 \\
12 & 0.468 & 0.649 \\
13 & 0.586 & 0.601 \\
14 & 0.696 & 0.707 \\
15 & 0.622 & 0.579 \\
16 & 0.750 & 0.643 \\
17 & 0.867 & 0.571 \\
18 & 0.909 & 0.477 \\
19 & 0.800 & 0.551 \\
20 & 1 & \\
\hline
\end{tabular}

discusses their health with whom; and, who shares their feelings with whom. Figure 7 displays the empirical distribution of edge-wise multiplexity indices (Koehly and Marcum, 2016; Smith et al., 2015) for each of these relations (in color), the four relations taken in aggregate (in gray), and among the four alter-alter and ego-alter relations (also in gray), respectively. Multiplexity is high across all four relationship types. Interestingly, alter-alter multiplexity is higher than ego-alter multiplexity. We investigated whether missingness or 'don't know' responses for alter-alter ties explained this result, but found only a trivial amount of evidence in favor of that hypothesis with only around $3 \%$ of the variation in alteralter multiplexity explained by structural problems associated with 'don't know' responses. Such a finding suggests that egos perceive their acquainted alters to be embedded in relatively homogeneous relationships with respect to network overlap; there was relatively consistent reporting across all relationship domains (e.g. if ego believes two alters share advice, ego likely also believes that those alters share feelings, etc.). Ego-alter ties, on the other hand, 
display lower multiplexity, suggesting that ego discriminates between relationship types for oneself. In other words, the lower ego-alter multiplexity can likely be attributed to egos differential knowledge about relations for oneself compared to how they perceive their alters' relationships with one another. 
Figure 7: Index of Multiplexity on Selected Relations

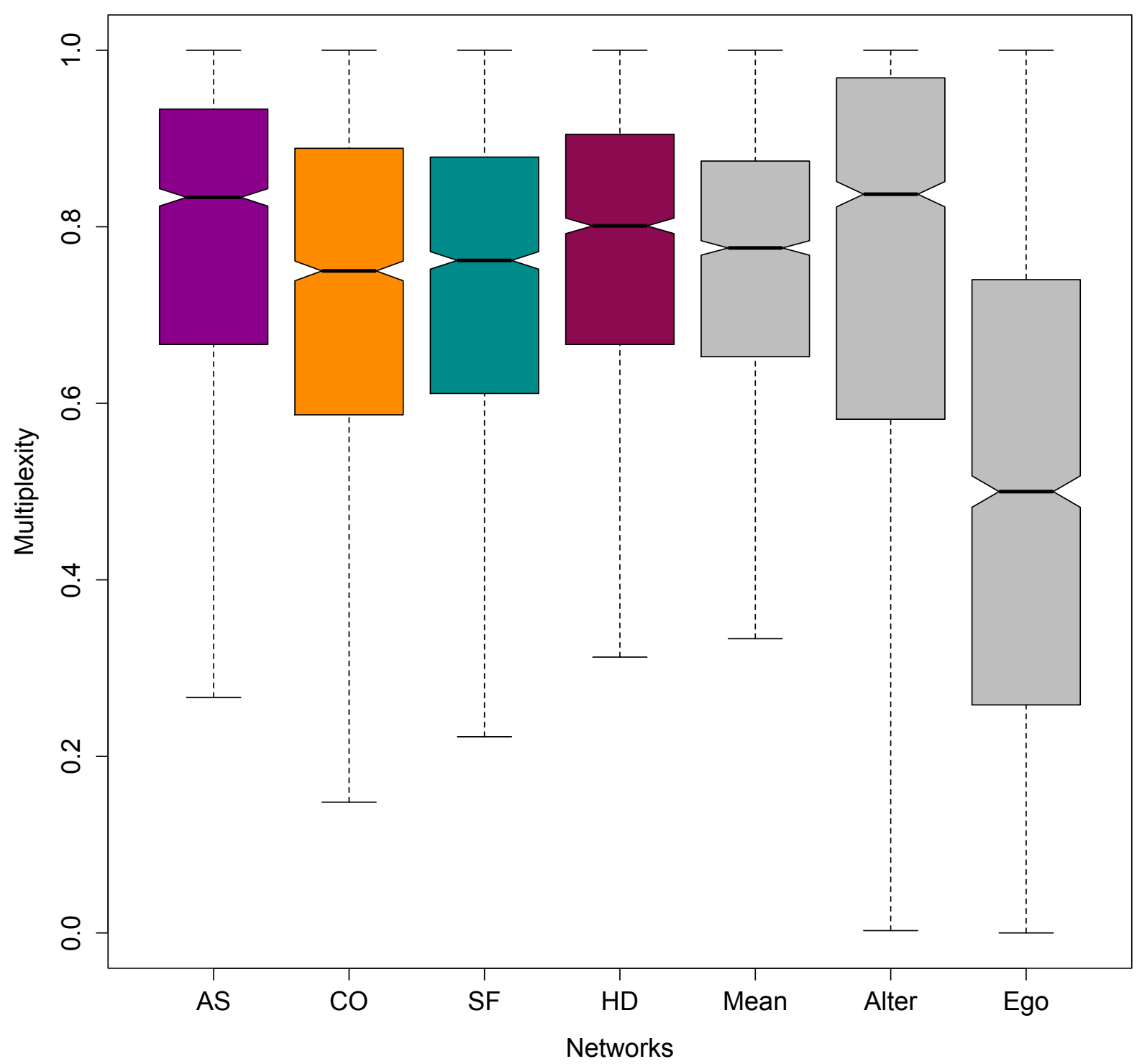

The first four colored boxplots represent the empirical distribution of edge-wise conditional multiplexity for Advice Seeking (AS), Consolation (CO), Seeking Favors (SF), and Health Discussion (HD), respectively. The remaining three gray boxplots represent the empirical distributions of the total average multiplexity, the average multiplexity only among alteralter ties, and the average multiplexity only among ego-alter ties, respectively. 


\section{Discussion}

Here, we provided definitional clarity to the term "personal networks" with a focus on four ego-centered network designs commonly employed in social network research. We show that the ego-centered cognitive social structure (ECSS) design is an effective strategy for collecting a large sample of network data in a cost-effective manner. ECSS networks represent the mental-maps used by individuals to organize and perhaps navigate their social worlds, thereby allowing investigation into how egos relate to their alters and how they perceive their alters to relate to one another. Although this design has been implemented before (Smith et al., 2015; Burt, 1984, among others), the current study is the first to formally distinguish its merits from other related sampling designs and to leverage the capabilities of online survey implementation to gather a large and representative sample of ECSS. The added benefit of using an online survey across a widespread geographic region permits independence assumptions in the sample, facilitates assessment of unlimited numbers of alters involved in multiple types of relationships, and makes feasible robust statistical inference at the population level.

The primary goal of this study was to implement the ECSS design on a large and representative scale. In doing so, we described perceptions of alter-alter ties by analyzing structural heterogeneity both within and between networks through examination of variation in network size, density, composition, structural correlation, and multiplexity. The results presented here provide a better understanding of the variability of close personal networks for the general population of the United States.

With respect to the scale and representativeness of our data, we drew upon previous literature that emphasizes the importance of kin and family on social ties (Thoits, 2011; Dunbar and Spoors, 1995). Incidentally, recent work has shown that there is a large amount of overlap between whom individuals count as friends and family (Bush et al., 2017), which resonates with our definition of close personal networks. Such network elicitation eases respondent burden to the extent that it maps onto heuristics already available to the partic- 
ipant (Brashears, 2013). Furthermore, we had a reasonable response rate of 53\%, indicating that there was not an undue likelihood of response bias in this sample, though we report evidence of respondent burnout in the largest networks.

Our results suggest that cognitive networks of close personal ties are heterogeneous in structure. First, there was sizeable variability in network size and density. The distribution of network size was heavily right-tailed, but had a median of about 9 alters and, consistent with past work, followed closely to a negative binomial candidate distribution (Handcock and Jones, 2004; Seeman et al., 2001). We note largely variant average density for the who-knowswho (WkW) network. Additionally, we observe denser subgroups at higher densities, which is in agreement with previous literature both on simulated (Friedkin, 1981) and empirical (Ter Wal, 2013) networks. In these data, the dense subgroups generally coincide with family and non-family clusters, indicating a cognitive separation of these groups, which likely reflects true clustering in relationships (but could also be reflective of the two enumeration prompts we asked respondents to fill out if respondents treated them separately). However, for the other networks, which are conditional on the WkW network, the densities of networks are largely homogeneous across network size. This may indicate that within clusters defined by the $\mathrm{WkW}$ network, egos tend to believe those clusters are similarly dense with respect to multiplex ties regardless of the overall size of one's cognitive network. This latter finding is important in that it shows people often think that those in their social networks interact with and relate to one another in similar ways, even if there is a large amount of variability in how many alters are in their social network and how they are perceived to be acquainted.

In addition to the insight garnered about heterogeneity in ECSS, we are also able to understand how participants recall and enumerate alters. As stated, we find that the distribution of number of alters is most accurately characterized by a negative binomial distribution. The negative binomial distribution is described by the number of successes one achieves until one has a certain number of failures. In this context, a success would be a suitable alter that the ego enumerates while a failure would be a recalled alter that the ego 
decides is not pertinent. After so many failures, the respondent stops trying to recall names. Alternatively, the negative binomial distribution can be composed of multiple Poisson processes. Given that we use two different network generators, the negative binomial may be the combination of independent Poisson processes from these generators; for each generator, respondents have, on average, a certain number of alters, and these independent processes result in the distribution we observe. We also observe evidence of binning in this distribution: substantially more people enumerated an even number of alters; this may be the result of affine dependence on recall (enumerating a friend may induce listing that friend's spouse, for example).

The multiplexity data also allow us to draw conclusions about the cognitive processes in the storage and recall of networks in the general population. We note that on average, health discussion and advice-seeking had higher multiplexity scores than did discussing feelings or consolation. This is likely because discussing health and seeking advice are indicative of more intimate relationships, suggesting that if a dyad engages in one of these ties, then it is likely that they also engage in the less intimate ties, but not vice versa. Less intuitive is the result that ego-alter multiplexity scores are generally lower than alter-alter multiplexity scores. One might expect that an ego enumerating their closest ties would have large amounts of multiplexity with those closest ties, whereas the alters may only minimally interact, reducing multiplexity. The simplest explanation for this is, again, affine dependence - people are intimately aware of their multiplex ties with their own alters, and would therefore be more discerning about different relationships. This would result in lower multiplexity. On the other hand, egos would not be as familiar with all aspects of various alter-alter ties. If however, they know that one or more of those ties do exist for a given alter-alter dyad, ego likely bins that tie across relationship types, which results in high multiplexity. This process is consistent with the network compression heuristics studied by Brashears (2013).

Our study had several limitations. First, although the financial cost is only mildly greater for ECSS than EEC, the cognitive burden on respondents is increased due to the questions 
about alter-alter ties. Instead of scaling linearly with the number of alters as in an EEC, ECSS scales closer to quadratic with the number of alters, even with our efforts to reduce how many alter-alter ties we interrogated. Additionally, the completely unconnected nature of our respondents means that the alter-alter ties enumerated are entirely cognitive; we have no way to verify them (e.g. by consensus) as in an multiple informant CSS. The severity of this problem depends on the research in question; inference about true relations may be biased, but if one is truly interested in how an individual perceives they are situated in their social network, then these data are ideal. Indeed, there are many instances where what one thinks of their social network is actually more important than the true structure for that individual (McDowell and Serovich, 2007). Additionally, the representative nature of the sample may be limited due to the fact that we collected via an online consumer panel. Thus, the results may be more reflective of the professional survey taking population than the population at large, despite the background characteristics of the sample matching well to the population). Finally, our enumerators were designed to elicit family members and non-family members separately: we did not examine the multiplexity of overlaps in network composition as others have recently done (Bush et al., 2017), which may be an important source of network heterogeneity. However, our focus on edgewise-conditional multiplexity highlighted differences in relational overlap net of any compositional overlaps per se.

Data such as these are ripe for exploration in a number of domains, and we outline a few potential extensions here. As mentioned, future work should entail understanding respondent fatigue in response to online surveys, and whether this might impact the resultant study population. Furthermore, we have described the characteristics of these networks as a whole and noted heterogeneity, but make no claims about the origins of this heterogeneity; future work should evaluate potential sources. For instance, recent work by Giannella and Fischer (2016) advanced a general typography of ego-centered networks; our knowledge of alter-alter ties could increase the discriminatory ability of such a method, as well as relate it to geography, given the spatially representative nature of the data. Herein, we also discussed 
plausible candidate cognitive processes that may have given rise to the data; however, we cannot test these proposed processes with the current data.

In conclusion, our results represent a substantial contribution to the study of ego-centered close personal networks. By eliciting both ego-alter and alter-alter ties from a large representative sample of the U.S. population our data provide the first generalizable empirical reference distribution for many network statistics on this subject. From this distribution, we've shown that even in relatively small networks (e.g., on the order of 9 alters), there is wide structural heterogeneity between networks. At the same time, we demonstrated that there was relatively high multiplexity within networks, on average. Taken together, these results suggest that future research on ego-centered networks may be better informed by between network than by within network comparisons at the population level. 


\section{References}

Ajrouch, K. J., Antonucci, T. C., and Janevic, M. R. (2001). Social networks among blacks and whites the interaction between race and age. The Journals of Gerontology Series B: Psychological Sciences and Social Sciences, 56(2):S112-S118.

Ashida, S. and Heaney, C. A. (2008). Differential associations of social support and social connectedness with structural features of social networks and the health status of older adults. Journal of Aging and Health, 20(7):872-893.

Berkman, L. F. and Syme, S. L. (1979). Social networks, host resistance, and mortality: a nine-year follow-up study of alameda county residents. American Journal of Epidemiology, $109(2): 186-204$.

Bernard, H. R., Johnsen, E. C., Killworth, P. D., McCarty, C., Shelley, G. A., and Robinson, S. (1990). Comparing four different methods for measuring personal social networks. Social Networks, 12(3):179-215.

Bernard, H. R., Johnsen, E. C., Killworth, P. D., and Robinson, S. (1991). Estimating the size of an average personal network and of an event subpopulation: Some empirical results. Social Science Research, 20(2):109-121.

Boster, J. S., Johnson, J. C., and Weller, S. C. (1987). Social position and shared knowledge: Actors' perceptions of status, role, and social structure. Social Networks, 9(4):375 - 387 .

Brashears, M. E. (2013). Humans use compression heuristics to improve the recall of social networks. Scientific Reports, 3.

Burt, R. S. (1984). Network items and the general social survey. Social Networks, 6(4):293 $-339$.

Bush, A. N., Walker, A. M., and Perry, B. L. (2017). "the framily plan": Characteristics 
of ties described as both "friend" and "family" in personal networks. Network Science, page 5 .

Butts, C. T. and Carley, K. M. (2005). Some simple algorithms for structural comparison. Computational $\&$ Mathematical Organization Theory, 11(4):291-305.

Campbell, K. E. and Lee, B. A. (1991). Name generators in surveys of personal networks. Social Networks, 13(3):203-221.

Cattell, V. (2001). Poor people, poor places, and poor health: the mediating role of social networks and social capital. Social Science \& Medicine, 52(10):1501-1516.

Christakis, N. A. and Fowler, J. H. (2007). The spread of obesity in a large social network over 32 years. New England Journal of Medicine, 357(4):370-379.

Dunbar, R. I. and Spoors, M. (1995). Social networks, support cliques, and kinship. Human Nature, 6(3):273-290.

Fischer, C. S. (1982). To Dwell Among Friends: Personal Networks in Town and City. University of Chicago Press.

Freeman, L. C. and Thompson, C. R. (1989). Estimating acquaintanceship volume. In Kochen, M., editor, The Small World, pages 147-158. Ablex, Norwood, NJ.

Friedkin, N. E. (1981). The development of structure in random networks: an analysis of the effects of increasing network density on five measures of structure. Social Networks, $3(1): 41-52$.

Giannella, E. and Fischer, C. S. (2016). An inductive typology of egocentric networks. Social Networks, 47:15-23.

Handcock, M. S. and Jones, J. H. (2004). Likelihood-based inference for stochastic models of sexual network formation. Theoretical Population Biology, 65(4):413-422. 
Kawachi, I., Subramanian, S. V., and Kim, D. (2008). Social capital and health. In Social capital and health, pages 1-26. Springer.

Killworth, P. D., McCarty, C., Johnsen, E. C., Bernard, H. R., and Shelley, G. A. (2006). Investigating the variation of personal network size under unknown error conditions. Sociological Methods \& Research, 35(1):84-112.

Klovdahl, A. S., Potterat, J. J., Woodhouse, D. E., Muth, J. B., Muth, S. Q., and Darrow, W. W. (1994). Social networks and infectious disease: The colorado springs study. Social Science $\mathscr{G}$ Medicine, 38(1):79-88.

Koehly, L. M., Ashida, S., Schafer, E. J., and Ludden, A. (2015). Caregiving networksusing a network approach to identify missed opportunities. The Journals of Gerontology Series B: Psychological Sciences and Social Sciences, 70(1):143-154.

Koehly, L. M. and Marcum, C. S. (2016). Multi-relational measurement for latent construct networks. Psychological Methods, 21(4):FirstView.

Koehly, L. M., Peterson, S. K., Watts, B. G., Kempf, K. K. G., Vernon, S. W., and Gritz, E. R. (2003). A social network analysis of communication about hereditary nonpolyposis colorectal cancer genetic testing and family functioning. Cancer Epidemiology Biomarkers 83 Prevention, 12(4):304-313.

Krackhardt, D. (1987). Cognitive social structures. Social Networks, 9(2):109-134.

Laumann, E. O., Marsden, P. V., and Prensky, D. (1989). The boundary specification problem in network analysis. In Freeman, L. C., White, D. R., and Romney, A. K., editors, Research Methods in Social Network Analysis, pages 61-87. George Mason University Press, Fairfax, VA.

Marsden, P. V. (1987). Core discussion networks of Americans. American Sociological Review, pages 122-131. 
Marsden, P. V. (1990). Network data and measurement. Annual Review of Sociology, 16(1):435-463.

Marsden, P. V. (2005). Recent Developments in Network Measurement, pages 8-30. Cambridge University Press, New York.

McCarty, C., Bernard, H. R., Killworth, P. D., Shelley, G. A., and Johnsen, E. C. (1997). Eliciting representative samples of personal networks. Social Networks, 19(4):303-323.

McCarty, C., Killworth, P. D., and Rennell, J. (2007). Impact of methods for reducing respondent burden on personal network structural measures. Social networks, 29(2):300315.

McDowell, T. L. and Serovich, J. (2007). The effect of perceived and actual social support on the mental health of hiv-positive persons. AIDS Care, 19(10):1223-1229.

McPherson, M., Smith-Lovin, L., and Brashears, M. E. (2006). Social isolation in America: Changes in core discussion networks over two decades. American Sociological Review, 71(3):353-375.

Milardo, R. M. (1992). Comparative methods for delineating social networks. Journal of Social and Personal Relationships, 9(3):447-461.

Morrison, E. W. (2002). Newcomers' relationships: The role of social network ties during socialization. The Academy of Management Journal, 45(6):1149-1160.

Neal, J. W. (2008). "kracking" the missing data problem: applying Krackhardt's cognitive social structures to school-based social networks. Sociology of Education, 81(2):140-162.

Newman, M. E. (2003). Properties of highly clustered networks. Physical Review E, 68(2):026121. 
Omalley, A. J., Arbesman, S., Steiger, D. M., Fowler, J. H., and Christakis, N. A. (2012). Egocentric social network structure, health, and pro-social behaviors in a national panel study of Americans. PLoS One, 7(5):e36250.

Roberts, S. G. and Dunbar, R. I. (2011). The costs of family and friends: an 18-month longitudinal study of relationship maintenance and decay. Evolution and Human Behavior, 32(3):186-197.

Roberts, S. G., Dunbar, R. I., Pollet, T. V., and Kuppens, T. (2009a). Exploring variation in active network size: Constraints and ego characteristics. Social Networks, 31(2):138-146.

Roberts, S. G., Dunbar, R. I., Pollet, T. V., and Kuppens, T. (2009b). Exploring variation in active network size: Constraints and ego characteristics. Social Networks, 31(2):138-146.

Seeman, T. E., Lusignolo, T. M., Albert, M., and Berkman, L. (2001). Social relationships, social support, and patterns of cognitive aging in healthy, high-functioning older adults: Macarthur studies of successful aging. Health Psychology, 20(4):243.

Shye, D., Mullooly, J. P., Freeborn, D. K., and Pope, C. R. (1995). Gender differences in the relationship between social network support and mortality: A longitudinal study of an elderly cohort. Social Science \&3 Medicine, 41(7):935-947.

Smith, E. J., Marcum, C. S., Boessen, A., Almquist, Z. W., Hipp, J. R., Nagle, N. N., and Butts, C. T. (2015). The relationship of age to personal network size, relational multiplexity, and proximity to alters in the western United States. The Journals of Gerontology Series B: Psychological Sciences and Social Sciences, 70(1):91-99.

Straits, B. C. (2000). Ego's important discussants or significant people: An experiment in varying the wording of personal network name generators. Social Networks, 22(2):123-140.

Ter Wal, A. L. (2013). The dynamics of the inventor network in German biotechnology: geographic proximity versus triadic closure. Journal of Economic Geography, pages 1-23. 
Thoits, P. A. (2011). Mechanisms linking social ties and support to physical and mental health. Journal of Health and Social Behavior, 52(2):145-161.

Väänänen, A., Buunk, B. P., Kivimäki, M., Pentti, J., and Vahtera, J. (2005). When it is better to give than to receive: long-term health effects of perceived reciprocity in support exchange. Journal of Personality and Social Psychology, 89(2):176.

van den Berg, P., Arentze, T., and Timmermans, H. (2009). Size and composition of egocentered social networks and their effect on geographic distance and contact frequency. Transportation Research Record: Journal of the Transportation Research Board, (2135):19 .

Van der Poel, M. G. (1993). Delineating personal support networks. Social Networks, 15(1):49-70.

Wasserman, S. and Faust, K. (1994). Social Network Analysis: Methods and Applications. Cambridge University Press, Cambridge.

Wellman, B. (1979). The community question: The intimate networks of east yorkers. American Journal of Sociology, 84(5):1201-1231.

Zheng, T., Salganik, M. J., and Gelman, A. (2006). How many people do you know in prison? using overdispersion in count data to estimate social structure in networks. Journal of the American Statistical Association, 101(474):409-423. 